\title{
IDENTIFICACIÓN Y EVALUACIÓN DE LOS COSTOS AMBIENTALES EN LA UNIDAD EMPRESARIAL DE BASE CENTRAL AZUCARERO “ARGEO MARTÍNEZ” DE GUANTÁNAMO
}

\section{IDENTIFICATION AND EVALUATION OF ENVIRONMENTAL COSTS IN THE BUSINESS UNIT OF SUGARCANE PRODUCTION CENTRAL BASE “ARGEO MARTÍNEZ” IN GUANTÁNAMO}

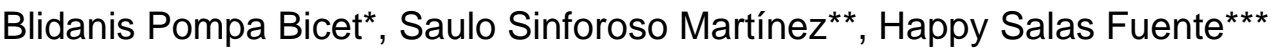

\footnotetext{
* Estudiante de Maestría, Profesora de la Facultad de Ciencias Económicas y Empresariales de la Universidad de Guantánamo, Cuba. Email: blidanispb@cug.co.cu. ORCID: https://orcid.org/0000-00015241-8220.
}

** Doctor en Ciencias Administrativas y Gestión para el Desarrollo, Profesor de Tiempo Completo de la Facultad de Contaduría de la Universidad Veracruzana, Región Poza Rica- Tuxpan, México. ORCID: https://orcid.org/0000-0001-6961-5546.

*** Doctor en Ciencias Contables, Profesor de la Facultad de Ciencias Económicas y Empresariales de la Universidad de Guantánamo, Cuba. Email: happysf@cug.co.cu. ORCID: https://orcid.org/0000-0001-93446623.

Dirección para recibir correspondencia: ssinforoso@uv.mx 
IDENTIFICACIÓN Y EVALUACIÓN DE LOS COSTOS AMBIENTALES EN LA UNIDAD EMPRESARIAL DE BASE CENTRAL AZUCARERO "ARGEO MARTÍNEZ" DE GUANTÁNAMO.

\section{RESUMEN}

OBJETIVO: Diseñar un procedimiento para el reconocimiento y evaluación de los costos ambientales en la UEB Central Azucarero "Argeo Martínez" de Guantánamo, que fortalezca el proceso de toma de decisiones.

MATERIAL Y METODO: En el desarrollo de éste, se emplearon un conjunto de métodos del nivel teórico y empírico que permiten validar la propuesta realizada. En este estudio, se aplica un procedimiento metodológico que permite el reconocimiento y evaluación de los costos ambientales en dicho sector.

RESULTADOS: El reconocimiento y posterior evaluación de los costos ambientales contribuye al fortalecimiento del proceso de toma de decisiones.

CONCLUSIÓN: Es imprescindible determinar cuánto le dedican las empresas al cuidado del medio ambiente, teniendo en cuenta que las entidades poseen un contrato implícito con la sociedad y el medio ambiente, producto de los recursos que utiliza y los desechos y desperdicios que genera.

PALABRAS CLAVE: Desarrollo sostenible. Costos ambientales. Impactos ambientales. Toma de decisiones. Industria azucarera.

\section{ABSTRACT}

OBJECTIVE: Design a procedure for the recognition and evaluation of environmental costs in the sugarcane production central base "Argeo Martínez" in Guantánamo (UEB that strengthens the decision-making process.

MATERIAL AND METHOD: In its development, a set of theoretical and empirical level methods were used to validate the proposal made. In this study a methodological procedure is applied that allows the recognition and evaluation of environmental costs in the mentioned sector.

RESULTS: The recognition and subsequent evaluation of environmental costs contribute to the strengthening of the decision-making process. 
IDENTIFICACIÓN Y EVALUACIÓN DE LOS COSTOS AMBIENTALES EN LA UNIDAD EMPRESARIAL DE BASE CENTRAL AZUCARERO "ARGEO MARTÍNEZ" DE GUANTÁNAMO.

CONCLUSION: It is essential to determine how much companies dedicate to care the environment, considering that the entities have an implicit contract with the society and the environment, because of the resources they use and the waste they generate.

KEY WORDS: Sustainable development. Environmental costs. Environmental impacts. Decision making. Sugar industry.

\section{MARCO TEÓRICO}

La gestión ambiental aborda la intención de alcanzar el equilibrio entre la demanda de recursos naturales y la capacidad de la naturaleza para dar respuesta a éstas, no sólo se identifica como problemas ambientales lo relativo a la contaminación y degradación del medio físico natural, sino relacionado con otras cuestiones sociales, culturales y económicas con estrecho vínculo al modelo de desarrollo, estos últimos juegan un papel determinante la gestión de los costos ambientales (Bandera, Otero y Ramos, 2017).

Los costos ambientales tienen antecedentes a nivel internacional desde el año 1948 (Sinforoso, Alvares y Vázquez, 2018). La gestión de los costos ambientales se convierte en un elemento imprescindible para alcanzar la sustentabilidad ambiental, al propiciar la conciliación de las actividades humanas (tanto económicas como sociales), con el medio ambiente (FerreiraSardinha, Pérez y Vilariño, 2018).

El principal problema de la Contabilidad de Gestión Medioambiental (CGMA) según la División de Naciones Unidas para el desarrollo sostenible (reconocida en inglés como Nations Division for Sustainable Development - UNDSD, 2001) es que se carece de una definición estándar de costo ambiental, ya que dependiendo de varios intereses, ellos incluyen una variedad de costos, por ejemplo, costo de disposición o costos de inversión y algunas veces, también costos externos por ejemplo costos incurridos fuera de la compañía, mayoritariamente hacia el público en general, por supuesto, esto también es cierto para las ganancias de actividades empresarias ambientales (ahorros de costos ambientales).

Los costos causados por los procesos extractivos, los vertimientos y residuos descargados al medio ambiente, resultado de las actividades humanas y de producción, no son representados 
IDENTIFICACIÓN Y EVALUACIÓN DE LOS COSTOS AMBIENTALES EN LA UNIDAD EMPRESARIAL DE BASE CENTRAL AZUCARERO "ARGEO MARTÍNEZ" DE GUANTÁNAMO.

por la contabilidad tradicional, o si se reconoce su existencia y valoración, se diluyen e invisibilizan por acumulación y su clasificación dentro de los costos tradicionales (Lemos y Rodríguez, 2016).

Adicionalmente, la mayoría de estos costos no se rastrean en forma sistemática ni se atribuyen a los procesos y productos responsables de los mismos, sumándolos simplemente en la estructura general, entonces se hace imprescindible saber identificarlos y clasificarlos, para esto aparece otra problemática, pues las clasificaciones de los costos ambientales son muy heterogéneas, existiendo una variedad de autores que brindan categorías distintas, pero hay que resaltar que los principios se mantienen, según el Documento No. 13 de la Asociación Española de Contabilidad y Administración de Empresas (AECA, 1996).

La definición de costos ambientales depende del horizonte de planeación empresarial y de la información requerida para el análisis. A continuación, se abordan algunas definiciones expuestas por diversas instituciones y autores entendidos en la temática.

Los costos ambientales son definidos por Hansen y Mowen (2007) como "aquellos en los que se incurre debido a que existe una calidad ambiental deficiente y que están asociados con la creación, la detección, el remedio y la prevención de la degradación ambiental". Estos costos se utilizan para medir el impacto ambiental causado por las actividades económicas de las organizaciones con la finalidad de tomar medidas de prevención.

Parodi (2010), afirma que los costos ambientales son un elemento más del costo de producción y tienen impacto en la fijación de los precios de venta. Salas (2015) en su definición considera que el costo económicamente es un sacrificio de recursos que es necesario realizar para poder elaborar un producto o servicio de cara a incrementar el valor añadido del mismo. Cuando el sacrificio que se realiza afecta a los recursos naturales y a la calidad de vida, se está en presencia de un costo ambiental; por lo tanto, estos son un elemento más del costo de producción y tienen impacto en la fijación de los precios de venta.

López (2019), afirma que un costo ambiental, está representado por todas las inversiones que realiza la empresa, en función al reconocimiento del impacto ambiental provocado como consecuencia de las actividades productivas y/o administrativas, cuyos resultados deben estar representados en los informes financieros. 
IDENTIFICACIÓN Y EVALUACIÓN DE LOS COSTOS AMBIENTALES EN LA UNIDAD EMPRESARIAL DE BASE CENTRAL AZUCARERO "ARGEO MARTÍNEZ" DE GUANTÁNAMO.

Por otra parte, Maura y Febles (2018) afirman que los costos ambientales constituyen el objeto principal de la contabilidad ambiental administrativa, y se definen como el resultado de las acciones $u$ omisiones que dañan el entorno. Al determinarlos, se previene, elimina, mitiga o rectifica el daño ambiental y se conserva el ambiente.

Noguera (2016), refiere cuando los esfuerzos económicos están orientados a la afectación de los recursos naturales y de calidad de vida, se está en presencia de costos ambientales y representan "la medida y valoración del esfuerzo por la aplicación racional de los factores ambientales de cara a la obtención de un producto, un trabajo o un servicio"

Los costos ambientales permiten a las empresas lograr una producción más eficiente en armonía con el medio ambiente, estas organizaciones logran certificaciones nacionales e internacionales, lo que afianza su posicionamiento en el mercado en donde se desarrollan. Para una toma acertada de decisiones gerenciales, los costos ambientales deberían ocupar un espacio único en la presentación de los estados financieros, así la administración de los recursos podrá realizar un análisis objetivo de las inversiones efectuadas y su impacto en la generación de la rentabilidad (Cueva, Martínez y Cobos, 2017).

Los costos ambientales no medidos ni identificados, permanecen ocultos dentro de los procesos de la organización y no pueden ser gestionados en sus características particulares. Se consideran costos, la inserción de los costos de medio ambiente y dentro del proceso de producción (Mastrapa y Sánchez, 2017).

En resumen, al considerar los elementos comunes y diferenciadores de las definiciones analizadas afirma que los "costos ambientales son los costos de las medidas emprendidas por una empresa; para prevenir, reducir y/o mitigar el deterioro ambiental como resultado de las actividades que realiza la empresa o para contribuir a la conservación de los recursos renovables y no renovables".

La identificación de los costos ambientales constituye una necesidad dentro del horizonte de planeación estratégica del sistema empresarial; sin embargo, es una realidad que los datos cuantitativos referidos al valor económico y social de la información de los mismos es escasa.

Como se plantea anteriormente, uno de los mayores problemas en la actualidad es la forma o la vía para poder identificar los costos ambientales, producto de que la contabilidad financiera no 
IDENTIFICACIÓN Y EVALUACIÓN DE LOS COSTOS AMBIENTALES EN LA UNIDAD EMPRESARIAL DE BASE CENTRAL AZUCARERO "ARGEO MARTÍNEZ" DE GUANTÁNAMO.

registra los costos ambientales estando estos incluidos dentro de algunas cuentas financieras. Además, las diferentes categorías que se utilizan dificultan el trabajo de los contadores, fundamentalmente por la superposición de conceptos (Becerra et al., 2011) y (Cañizares, 2015).

Una compañía define un costo ambiental según como se propone utilizar la información (asignación de coste, presupuesto de capital, diseño de procesos / productos, otras decisiones de gerencia), así como la escala y el alcance del ejercicio. Por otra parte, puede no estar claro si un costo es ambiental o no; algunos costos bajan en una zona gris o se pueden clasificar como en parte ambiental y en parte no. En sentido general, los costos ambientales pueden ser agrupados en cuatro grandes categorías que se definen a continuación:

Costos de prevención: Es el costo de las actividades que se llevan a cabo para evitar la producción de contaminantes y/o desechos que pueden dañar el medioambiente.

Costo de detección: Aquellos en los que se incurren para determinar si los productos, procesos o actividades son acordes a los estándares ambientales.

Costo por fallas internas: Ocurren cuando se realizan actividades que producen desperdicios contaminantes pero que aún no han sido vertidos al ambiente.

Costos por fallas externas: Son los costos que se generan después son sacados de la empresa. Estos a su vez pueden subdividirse en realizados (incurridos realmente por la empresa) y no realizados (determinados costos de la sociedad, consecuencia de la empresa, que de alguna manera afectan a la sociedad).

A modo de resumen, se aprecia que en la actualidad no existe un consenso en cuanto a la forma más adecuada de identificar los costos ambientales, pero la revisión efectuada confirma que constituye una necesidad el reconocimiento y evaluación de los mismos para perfeccionar el sistema de información contable, la evaluación del desempeño empresarial y el proceso de toma de decisiones. Por otra parte, con el reconocimiento de los costos ambientales se genera una información financiera completa, buscando beneficios empresariales y ambientales.

Ante un mercado donde el tema ambiental es prioritario, se concluye que el costo ambiental se convierte en una ventaja competitiva para las organizaciones, los responsables de las empresas tendrán información que les permitirá identificar los impactos que su operatividad ocasiona al 
IDENTIFICACIÓN Y EVALUACIÓN DE LOS COSTOS AMBIENTALES EN LA UNIDAD EMPRESARIAL DE BASE CENTRAL AZUCARERO "ARGEO MARTÍNEZ" DE GUANTÁNAMO.

medio ambiente, lo que facilita la toma de decisiones. Una entidad económica que carece de información en materia ambiental está destinada al fracaso, pues no tendría las bases para implementar acciones eficaces y eficientes en beneficio de los recursos naturales (Sinforoso 2018, p. 155).

\section{MATERIAL Y MÉTODO}

Los elementos abordados anteriormente, constituyen la base para la propuesta realizada por los autores de la presente investigación, basada en un procedimiento que tiene como propósitos principales, primero dotar a la empresa de los criterios generales que le permita reconocer en su sistema de información contable los costos ambientales incurridos en la aplicación de su política ambiental y de una serie de indicadores que posibiliten la evaluación de forma oportuna y adecuada del desempeño y la gestión empresarial y segundo, satisfacer la actual demanda de información contable ambiental de los diferentes grupos de usuarios.

El reconocimiento de los costos ambientales que fortalezca el proceso de toma de decisiones, se sustenta en un procedimiento conformado por tres pasos, como se aprecia en la (ver figura 1); además de declararse los objetivos específicos, técnicas y las salidas que se prevén obtener. Constituye una premisa para su aplicación conveniente, pero no excluyente, tener implementado el Sistema de Gestión Ambiental (SGA), para que aquellas empresas que no lo poseen puedan incorporarlo en la misma medida en que avancen en la implementación del sistema.

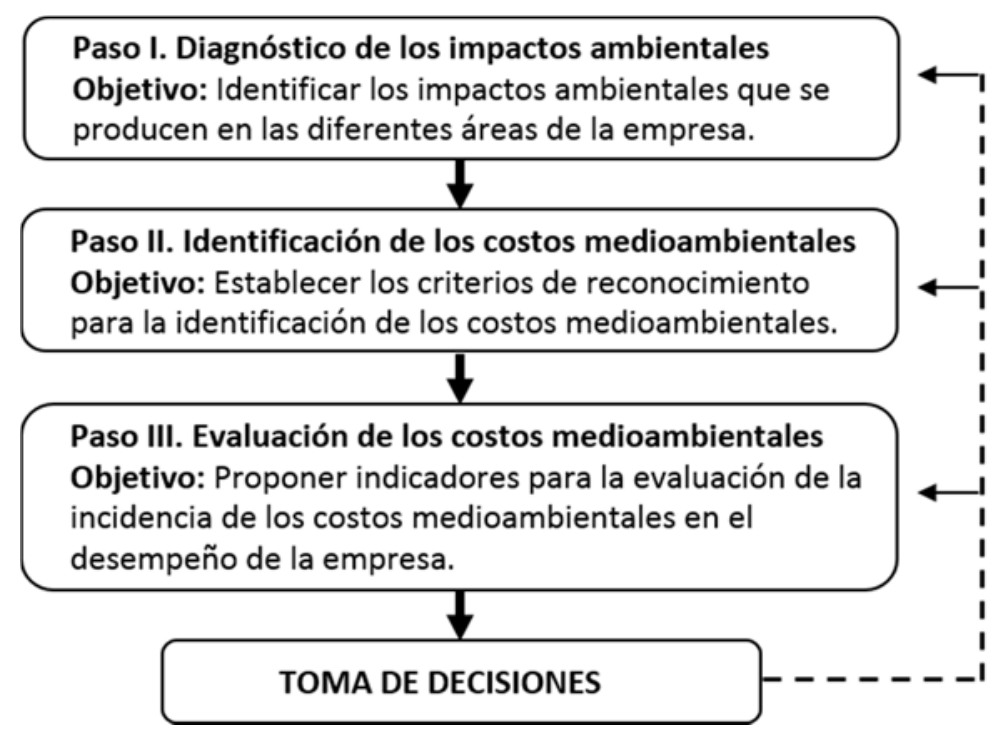

Figura 1. Procedimiento para el reconocimiento y evaluación de los costos medioambientales.

Fuente: Elaboración propia. 
IDENTIFICACIÓN Y EVALUACIÓN DE LOS COSTOS AMBIENTALES EN LA UNIDAD EMPRESARIAL DE BASE CENTRAL AZUCARERO "ARGEO MARTÍNEZ" DE GUANTÁNAMO.

\section{Paso I.- Diagnóstico de los impactos ambientales}

Objetivo: identificar los impactos ambientales que se producen en las diferentes áreas de la empresa.

Técnicas: revisión documental, observación, entrevista y dinámica grupal.

Un aspecto ambiental es "un elemento de las actividades, productos o servicios de una organización que puede interactuar con el medio ambiente" (NC ISO 14004:2004). Por ende, para la identificación de un aspecto ambiental se tendrán en cuenta los siguientes elementos:

$\checkmark$ Resultados del diagnóstico ambiental de cada área.

$\checkmark$ Requisitos legales aplicables a la organización.

$\checkmark$ Criterios de desempeño interno que se ha propuesto la entidad.

$\checkmark$ Diagrama de flujo de los procesos.

$\checkmark$ Características de los productos utilizados por la organización.

$\checkmark$ Tecnologías que se utilizan para realizar los procesos y estado técnico de los equipos.

$\checkmark$ Esquema general de los conductos, canalizaciones y drenajes que hay en cada instalación.

$\checkmark$ Diagrama del trasiego de los desechos sólidos y líquidos en cada instalación y su disposición.

$\checkmark$ Cantidad de desechos peligrosos que se generan y clasificación de los mismos.

$\checkmark$ Cantidad de fuentes de emisiones al aire, descargas al agua y a los suelos.

$\checkmark$ Cantidad de gases por fuente, caracterización de estos y descripción de los sistemas de extracción.

$\checkmark$ Consumo de materias primas y de energía para cada actividad.

$\checkmark$ Recursos naturales que se utilizan en las actividades y consumo de los mismos.

$\checkmark$ Verificar los índices de consumo según las normas técnicas establecidas para cada proceso, producto, servicio o tecnología de producción.

$\checkmark$ Si en algún proceso hay sobreconsumos se deben determinar las causas.

$\checkmark$ Estado actual de los almacenes, cumplimiento de las normas técnicas de almacenamiento para las materias primas y rotación de estas.

$\checkmark$ Competencia del personal en materia de medio ambiente.

En cada proceso se determinarán las características de los equipos e instalaciones, las materias primas utilizadas, las etapas por las cuales transita el proceso y el uso de recursos naturales tales como el agua. Igualmente se determinarán los productos resultantes de la realización del proceso POMPA-BICET B., SINFOROSO-MARTÍNEZ S., SALAS-FUENTE H. 
IDENTIFICACIÓN Y EVALUACIÓN DE LOS COSTOS AMBIENTALES EN LA UNIDAD EMPRESARIAL DE BASE CENTRAL AZUCARERO "ARGEO MARTÍNEZ" DE GUANTÁNAMO.

y los residuos que se generan del mismo, tales como: efluentes líquidos, emisiones al aire, derrames.

Para la identificación de los aspectos ambientales en las áreas, se podrán utilizar técnicas, tales como: observación de los procesos, análisis de contenido y entrevistas. Luego de determinar los aspectos ambientales de cada área, estos serán plasmados en el listado de aspectos e impactos ambientales.

Este listado debe brindar información detallada sobre los aspectos ambientales presentes en cada área que se genera, y así como el proceso del sistema asociado. Culminado el proceso de identificación de aspectos ambientales, se procederá a determinar los impactos ambientales, que constituyen cualquier cambio en el medioambiente, sea adverso o beneficioso, total o parcialmente resultante de las actividades, productos o servicios de una organización" (NC ISO 14004:2004) los cuales se plasmarán en el listado de aspectos e impactos ambientales.

Para cada aspecto e impacto ambiental se procederá a determinar su Nivel de significancia (S). El (S) se calcula multiplicando las puntuaciones obtenidas para la frecuencia con que ocurra el aspecto, la probabilidad de ocurrencia del impacto y la gravedad del mismo.

$$
\mathrm{S}=\mathrm{F}^{*} \mathrm{P}^{*} \mathrm{G}
$$

Donde:

$\mathrm{S}=$ Nivel de significancia

$\mathrm{F}=$ Frecuencia con que ocurre el aspecto

$\mathrm{P}=$ Probabilidad de que ocurra el impacto

$\mathrm{G}=$ Posible gravedad del impacto producido

Se considera un aspecto ambiental significativo si: $S>100$, así todos los aspectos ambientales que obtengan un nivel de significancia mayor que 100, serán significativos y serán prioritarios a la hora de establecer objetivos, metas y programas ambientales.

La identificación y valoración de los aspectos ambientales e impactos significativos posee una importancia de primer orden, se considera una de las bases decisivas sobre la cual se debe implementar el SGA. Los problemas que no se detecten en esta etapa, quedarían fuera de POMPA-BICET B., SINFOROSO-MARTÍNEZ S., SALAS-FUENTE H. 
IDENTIFICACIÓN Y EVALUACIÓN DE LOS COSTOS AMBIENTALES EN LA UNIDAD EMPRESARIAL DE BASE CENTRAL AZUCARERO "ARGEO MARTÍNEZ" DE GUANTÁNAMO.

cualquier análisis posterior y consecuentemente fuera del alcance del SGA que se proyecta y pueden poner en peligro los efectos deseados del mismo.

\section{Paso II.- Identificación de los costos ambientales}

Objetivo: establecer los criterios de reconocimiento para la identificación de los costos ambientales.

Técnicas: revisión documental y dinámica grupal.

Una vez identificados los aspectos e impactos ambientales de las diferentes áreas de la empresa, se procede a establecer los criterios de reconocimiento de los costos ambientales que se originan en las mismas con el propósito de cumplir con los objetivos y las metas planificados en la política ambiental de la entidad.

Para la clasificación de los costos ambientales, los autores asumen el criterio dado por Sánchez y Bifaretti (2008), sobre costos ambientales normales o de explotación, costos ambientales de evaluación y costos ambientales de control, partiendo de la incorporación del recurso al proceso de que se trate:

$\checkmark$ Costos Normales o de Explotación: son los asociados directamente con los productos y se utilizan como base para asignar la mano de obra directa en los costos de producción.

$\checkmark$ Costos de Evaluación: son los que se ocupan de medir y monitorear las fuentes potenciales de daños ambientales.

$\checkmark$ Costos de Control: son aquellos dirigidos a contener sustancias peligrosas que son utilizadas o producidas.

El establecimiento de estos criterios está encaminado a diferenciar entre los costos de las actividades normales de los que se relacionan con la conservación y cuidado del medio ambiente, los cuales son utilizados como variables esenciales en el diseño de los indicadores económicos y ambientales. De esta forma, se contribuye al control y uso eficiente de los recursos naturales y se fortalece el proceso de toma de decisiones. 
IDENTIFICACIÓN Y EVALUACIÓN DE LOS COSTOS AMBIENTALES EN LA UNIDAD EMPRESARIAL DE BASE CENTRAL AZUCARERO "ARGEO MARTÍNEZ" DE GUANTÁNAMO.

\section{Paso III.- Evaluación de los costos ambientales}

Objetivo: proponer indicadores para la evaluación de la incidencia de los costos ambientales en el desempeño de la empresa.

Técnicas: dinámica grupal.

Para realizar la evaluación de los costos ambientales de la empresa, la mejor forma es saber cómo se comportan estos en relación a otros parámetros de importancia en los análisis económicos financieros de la organización. El diseño de los indicadores se realizó mediante una dinámica grupal con un conjunto de especialistas con vasta experiencia en la actividad económica y ambiental. Los indicadores resultantes de la técnica aplicada se detallan a continuación:

1.- Peso específico de los Costos Ambientales Normales o de Explotación (PeCMN).

Dónde:

$\mathrm{CMN}=$ Costos Ambientales Normales o de Explotación

$$
\mathrm{CMAt}=\text { Costos Ambientales Totales }
$$

Interpretación: expresa la correlación existente entre los costos de los ambientales normales o de explotación por cada peso del costo ambiental total o el porciento que representan los CMN del CMAt.

2.- Peso específico de los Costos Ambientales de Evaluación (PeCMEv).

Donde:

$\mathrm{CMEv}=$ Costos Ambientales de Evaluación

CMAt $=$ Costos Ambientales Totales

Interpretación: expresa la relación existente entre los costos ambientales de evaluación por cada peso del costo ambiental total o el porciento que representan los CMEv del CMAt. 
IDENTIFICACIÓN Y EVALUACIÓN DE LOS COSTOS AMBIENTALES EN LA UNIDAD EMPRESARIAL DE BASE CENTRAL AZUCARERO "ARGEO MARTÍNEZ" DE GUANTÁNAMO.

3.- Peso específico de los Costos Ambientales de Control (PeCMCont).

Donde:

$$
\begin{aligned}
\text { CMAt } & =\text { Costos Ambientales de Control } \\
\mathrm{CT} & =\text { Costos Ambientales Totales }
\end{aligned}
$$

Interpretación: expresa la correlación existente entre los costos ambientales de control por cada peso del costo ambiental total o el porciento que representan los CMCont del CMAT.

4.- Peso específico de los Costos Ambientales Totales (PeCMAt).

Donde:

$$
\begin{gathered}
\text { CMAt }=\text { Costos Ambientales Totales } \\
\text { CT }=\text { Costos Totales }
\end{gathered}
$$

Interpretación: expresa la proporción existente entre los costos ambientales totales por cada peso del costo total o el porciento que representan los CMAt del CT.

5.- Costos Ambientales Totales por peso de ventas (CMAtxV).

Donde:

$$
\begin{gathered}
\text { CMAt }=\text { Costos Ambientales Totales } \\
\qquad n=\text { Ventas netas }
\end{gathered}
$$

Interpretación: expresa la correlación existente entre los costos ambientales totales por cada peso de ventas netas. Representa cuanto es sufragado de los CMAt por cada peso de venta.

\section{RESULTADOS}

\section{Paso I.- Diagnóstico de los impactos ambientales}

Mediante la revisión documental, la observación de los procesos de "Argeo Martínez" y la realización de una dinámica grupal con trabajadores, directivos y especialistas de la empresa, se 
IDENTIFICACIÓN Y EVALUACIÓN DE LOS COSTOS AMBIENTALES EN LA UNIDAD EMPRESARIAL DE BASE CENTRAL AZUCARERO "ARGEO MARTÍNEZ" DE GUANTÁNAMO.

identificaron los aspectos e impactos ambientales de las diferentes actividades y se obtuvo el siguiente resultado:

Los procesos de realización del producto son los que más influyen de manera perjudicial en el medio ambiente.

Las actividades que más impactan en el medio ambiente son: elaboración de productos y gestión de la infraestructura y el medio ambiente de trabajo.

$\checkmark$ Se identifican un total de 16 aspectos ambientales, de ellos los más significativos son: vertimiento de residuales líquidos, vertimiento de residuos sólidos y desechos peligrosos (bagazo, cogollos, vainas, hojas y trozos de cañas), pérdida de la diversidad biológica, emisiones de ruido y proliferación de olores desagradables.

$\checkmark$ Se evidencian un total de 20 impactos, de ellos los 20 resultaron significativos, que implican alteraciones, principalmente en los componentes ambientales agua, suelo y atmósfera, expresados a través de la contaminación al suelo, las aguas superficiales y subterráneas (por vertimiento de residuales líquidos, generación de residuos sólidos y desechos peligrosos y derrame de combustibles) y contaminación a la atmósfera (por olores desagradables y el ruido), además son considerables los riesgos y afectaciones reales a la salud humana .

Una vez listados los aspectos e impactos ambientales que producen las distintas áreas de "Argeo Martínez", se procede al reconocimiento de los costos medioambientales a través de la aplicación de los criterios definidos en el procedimiento.

\section{Paso Il.- Identificación de los costos ambientales}

La tabla 1, evidencia que en la empresa objeto de estudio se experimenta un incremento de los costos ambientales en el año 2018 con relación al 2017 de \$9932,149.34, motivado por el aumento de los costos normales o de explotación en $\$ 9^{\prime} 932,149.34$, manteniéndose en $\$ 43$, 637.83 los costos de control y en 0.00 los costos de evaluación.

Esta situación es desfavorable para la entidad, toda vez que demuestra que aún y cuando se implementan las medidas contempladas en su plan de manejo y de la política ambiental, hay que destacar de forma particular que el resultado es desfavorable, debido a que en el año 2018, los costos normales o de explotación crecen en mayor proporción que los costos de control. 
IDENTIFICACIÓN Y EVALUACIÓN DE LOS COSTOS AMBIENTALES EN LA UNIDAD EMPRESARIAL DE BASE CENTRAL AZUCARERO "ARGEO MARTÍNEZ" DE GUANTÁNAMO.

Es por ello, que la UEB Central Azucarero "Argeo Martínez" debe encaminar acciones dirigidas a que su gestión ambiental tenga un carácter preventivo, con lo cual podrá reducir su impacto y de esta forma evitar las posibles pérdidas por daños ocasionados al medio ambiente y el incremento de los costos.

\section{Tabla 1}

Reconocimiento de los costos medioambientales en "Argeo Martínez"

\begin{tabular}{|c|c|c|c|c|}
\hline Clasificación & Indicador & UM & 2018 & 2017 \\
\hline & Equipos & $\$$ & $43^{\prime} 000,195.80$ & $35^{\prime} 106,413.38$ \\
\hline $\begin{array}{l}\text { Costos } \\
\text { medioambientales }\end{array}$ & Materiales & $\$$ & $36^{\prime} 219,404.30$ & $33^{\prime} 811,634.92$ \\
\hline \multirow{2}{*}{$\begin{array}{l}\text { Normales o de } \\
\text { explotación }\end{array}$} & Mano de Obra Directa & $\$$ & $3 ’ 843,178.33$ & $4^{\prime} 212,580.79$ \\
\hline & Total & $\$$ & $83^{\prime} 062,778.43$ & $73^{\prime} 130,629.09$ \\
\hline \multirow[t]{3}{*}{ Costos de Evaluación } & & $\$$ & 0.00 & 0.00 \\
\hline & Planta de tratamiento & $\$$ & $30 ` 589.83$ & $30^{\prime} 589.83$ \\
\hline & Tanques reforzados para & & & \\
\hline \multirow[t]{2}{*}{ Costos de Control } & $\begin{array}{l}\text { almacenar productos } \\
\text { químicos }\end{array}$ & $\$$ & $13^{\prime} 048.00$ & $13^{\prime} 048.00$ \\
\hline & Total & $\$$ & $43,637.83$ & $43,637.83$ \\
\hline \multicolumn{2}{|c|}{ Total de costos Medioambientales } & $\$$ & $83^{\prime} 106,416.26$ & $73^{\prime} 174,266.92$ \\
\hline
\end{tabular}

Fuente: Elaboración propia a partir de los criterios de reconocimiento aplicados.

\section{Paso III.- Evaluación de los costos ambientales}

En la tabla 2, se observa que en el año 2018 los costos ambientales normales o de explotación representan el $99.94 \%$ del total de costos ambientales totales; es decir, por cada peso de costos ambientales totales la empresa incurre en \$ 0.9994 de costos ambientales normales o de explotación, mientras que en el año 2017 los costos ambientales normales o de explotación 
IDENTIFICACIÓN Y EVALUACIÓN DE LOS COSTOS AMBIENTALES EN LA UNIDAD EMPRESARIAL DE BASE CENTRAL AZUCARERO "ARGEO MARTÍNEZ" DE GUANTÁNAMO.

representan el $99.94 \%$ del total de costos ambientales totales, o sea, por cada peso de costos ambientales totales, \$ 0.9994 están asociados a costos ambientales normales o de explotación.

Tabla 2

Peso específico de los Costos Ambientales Normales o de Explotación (PeCMN)

\begin{tabular}{lcccc}
\hline Indicadores & UM & 2018 & 2017 & Variación \\
\hline $\begin{array}{l}\text { Costos ambientales normales o de } \\
\text { explotación }\end{array}$ & $\$$ & $83^{\prime}$ 062, 778.43 & $73^{\prime} 130,629.09$ & $9^{\prime}$ 932, 149.34 \\
$\begin{array}{lcccc}\text { Costos medioambientales totales } \\
\text { Resultados }\end{array}$ & $\$$ & $83^{\prime} 106,416.26$ & $73^{\prime} 174,266.92$ & $9^{\prime} 932,149.34$ \\
\hline
\end{tabular}

Fuente: Elaboración propia a partir de los cálculos realizados.

En la tabla 3, se aprecia que en el año 2018 los costos ambientales de control representan el $5.2 \%$ del total de costos ambientales totales; es decir, por cada peso de costos ambientales totales la empresa incurre en \$ 0.052 de costos ambientales de control, mientras que en el año 2017 los costos ambientales de control representan el 5.9\% del total de costos ambientales totales; o sea, por cada peso de costos ambientales totales, \$ 0.059 están asociados a costos ambientales de control. Esta situación es desfavorable para ambos años, ya que los costos de control deben aumentar.

Tabla 3

Peso específico de los Costos Ambientales de Control (PeCMCont)

\begin{tabular}{lcccc}
\hline Indicadores & UM & 2018 & 2017 & Variación \\
\hline Costos medioambientales de control & $\$$ & $43,637.83$ & $43,637.83$ & - \\
Costos medioambientales totales & $\$$ & $83^{\prime} 106,416.26$ & $73^{\prime} 174,266.92$ & $9^{\prime} 932,149.34$ \\
Resultados & & 0.052 & 0.059 & 0.00 \\
\hline
\end{tabular}

Fuente: Elaboración propia a partir de los cálculos realizados.

Como se observa en la tabla 4, los costos ambientales totales representan el $224,4 \%$ del total de costos de "Argeo Martínez", producto al incremento de los costos ambientales normales o de explotación; de forma general la situación es favorable para la entidad porque los costos ambientales tienen un peso significativo en la conformación del costo total; sin embargo, de manera específica este resultado es desfavorable producto a que en los costos ambientales 
IDENTIFICACIÓN Y EVALUACIÓN DE LOS COSTOS AMBIENTALES EN LA UNIDAD EMPRESARIAL DE BASE CENTRAL AZUCARERO "ARGEO MARTÍNEZ" DE GUANTÁNAMO.

normales o de explotación los conceptos que crecen no están asociados a la gestión de residuos, emisiones y vertimiento de productos, entre otros.

Tabla 4

Peso específico de los Costos Ambientales Totales (PeCMAt)

\begin{tabular}{lcccc}
\hline Indicadores & UM & 2018 & 2017 & Variación \\
\hline $\begin{array}{l}\text { Costos medioambientales } \\
\text { totales }\end{array}$ & $\$$ & $83^{\prime} 106,416.26$ & $73^{\prime} 174,266.92$ & $9^{\prime} 932,149.34$ \\
$\begin{array}{l}\text { Costos totales } \\
\text { Resultados }\end{array}$ & $\$$ & $37^{\prime}$ 032, 389.44 & $34^{\prime} 468,887.15$ & $2^{\prime} 563,502.29$ \\
\hline
\end{tabular}

Fuente: Elaboración propia a partir de los cálculos realizados.

En la tabla 5, se identifica que en el año 2018 los costos ambientales totales representan el $194.7 \%$ de las ventas netas, es decir, la unidad por cada peso de ventas netas incurre en \$ 1.947 de costos ambientales totales. Este indicador disminuye comparado con el año 2017, ya que los costos ambientales totales constituyen el $198.2 \%$ de las ventas netas.

Tabla 5

Costos Ambientales Totales por peso de ventas (CMAtxV)

\begin{tabular}{lcccc}
\hline Indicadores & UM & 2018 & 2017 & Variación \\
\hline $\begin{array}{l}\text { Costos medioambientales } \\
\text { totales }\end{array}$ & $\$$ & $83^{\prime} 106,416.26$ & $73^{\prime} 174,266.92$ & $9^{\prime} 932,149.34$ \\
$\begin{array}{l}\text { Ventas Netas } \\
\text { Resultados }\end{array}$ & $\$$ & $42^{\prime} 676,353.82$ & $36^{\prime} 913,104.18$ & $5^{\prime} 763,249.64$ \\
\hline
\end{tabular}

Fuente: Elaboración propia a partir de los cálculos realizados.

\section{CONCLUSIONES}

La industria azucarera es una de las que provoca mayor impacto negativo sobre el medio ambiente, ya que ella genera emisiones de gases efecto invernadero, altas emisiones de residuos con una elevada demanda química de oxígeno, así como una gran incidencia sobre el suelo, originado por los métodos productivos y de cosecha de la caña. El diagnóstico realizado en la investigación deja ver, que el marco normativo vigente, muestra aún dificultades en el reconocimiento y evaluación de los costos ambientales en el sistema de información contable de las empresas del sector azucarero. 
IDENTIFICACIÓN Y EVALUACIÓN DE LOS COSTOS AMBIENTALES EN LA UNIDAD EMPRESARIAL DE BASE CENTRAL AZUCARERO "ARGEO MARTÍNEZ" DE GUANTÁNAMO.

La Contabilidad Ambiental debe continuar el perfeccionamiento de su marco conceptual y normativo, para instituirse como un instrumento que suministre información útil y relevante y además que fortalezca el proceso de toma de decisiones. Los costos ambientales deben ser razonados como un mecanismo de prevención, control y mitigación que no solo sea amparado por la parte financiera de la organización, sino también como concientización a nivel organizacional a cerca de los procesos que se están desarrollando y de alguna u otra forma, generar procesos que proporcionen un aprovechamiento económico sostenible.

Los elementos teóricos, metodológicos y prácticos analizados en la investigación, permiten corroborar que el empleo de la Contabilidad de Gestión Ambiental y dentro de esta, el tratamiento de los costos ambientales, constituyen una necesidad para el fortalecimiento de la toma de decisiones en la industria Azucarera. El procedimiento propuesto se caracteriza por su flexibilidad, adaptabilidad y sencillez, cualidades que posibilitan su aplicación y generalización en otras entidades del sector. Los criterios para el reconocimiento de los costos ambientales y la aplicación de los indicadores propuestos favorecen el fortalecimiento de los SGA y la formulación de políticas para la toma de decisiones en la UEB Central Azucarero "Argeo Martínez".

\section{RECOMENDACIONES}

Se recomienda generalizar el algoritmo metodológico y los resultados investigativos obtenidos para el reconocimiento y evaluación de los costos ambientales en empresas afines al sector para el fortalecimiento del proceso de toma de decisiones. Asimismo, crear e implementar un SGA, que permita cumplir con las leyes y regulaciones ambientales, así como el desarrollo de una cultura ambiental en los trabajadores y directivos.

Otra recomendación es incorporar los indicadores propuestos en el SGA, las memorias descriptivas de los estados financieros, así como en los informes económicos y de gestión para el fortalecimiento de los criterios de evaluación de la gestión y el desempeño ambiental empresarial de la Azucarera Guantánamo. Por último, capacitar al personal de la empresa Azucarera Guantánamo con los nuevos conceptos expuestos en la investigación para lograr un mayor entendimiento de los mismos. 
IDENTIFICACIÓN Y EVALUACIÓN DE LOS COSTOS AMBIENTALES EN LA UNIDAD EMPRESARIAL DE BASE CENTRAL AZUCARERO "ARGEO MARTÍNEZ" DE GUANTÁNAMO.

\section{REFERENCIAS BIBLIOGRÁFICAS}

Albuerne, M. y Venereo, N. (2017). La contabilidad y la actividad medio ambiental de la industria ronera en Cuba: caso de estudio empresa mixta Havana Club International S.A-Ronera San José. Contabilidad y Negocios, 12(24), 1-14. Recuperado de http://www.redalyc.org/articulo.oa?id=281654984002

Asociación Española de Contabilidad y Administración de Empresas. (1996). Contabilidad de gestión en Latinoamérica. Ediciones AIC-AECA: España. Recuperado de http://www.observatorio-iberoamericano.org/Libro\%20\%20La\%20contab\%20de\%20gesti\%C3\%B3n\%20en\%20Latinoam\%C3\%A9rica/Espa\%C3 $\%$ B1a.htm

Bandera, Y., Otero, J. y Ramos, S. (2017). Gestión de costos ambientales en las exportaciones del Grupo Empresarial LABIOFAM: Papel del capital humano. En R. Velázquez (Presidencia), Conferencia Científica Internacional. Conferencia llevada a cabo en la Universidad de Holguín, Cuba. Recuperado de https://eventos.uho.edu.cu/index.php/ccm/cci2017/paper/viewFile/2255/839

Becerra, K., Gómez, E., Pérez, G. y Reyes, R. (2011). ¿Cómo calcular los costos medioambientales? Caso: Empresa Gráfica de Cienfuegos. Visión de Futuro, 15(2), 1-23. Recuperado de https://revistacientifica.fce.unam.edu.ar/index.php?option=com_content\&view=article\&id=2 65:icomo-calcular-los-costos-medioambientales-caso-empresa-grafica-decienfuegos\&catid=105: articulos-en-espanol

Cárdenas, P., Ore, K., Vera, C. (2016). Los costos ambientales y su efecto en la gestión gerencial de las empresas agroexportadoras de espárragos (Tesis de pregrado). Universidad Peruana de Ciencias Aplicadas, Lima, Perú. Recuperado de https://repositorioacademico.upc.edu.pe/bitstream/handle/10757/621455/ORE_QK\%20\%2 0\%20CARDENAS\%20_RP\%20\%20\%20VERA_VC.pdf?sequence=2\&isAllowed=y

Cañizares, M. (2015). Procedimiento para cuantificar los costos de las actividades ambientales en la gestión de sostenibilidad del recurso agua potable (Tesis doctoral). Universidad de La Habana, La Habana. Cuba. 
IDENTIFICACIÓN Y EVALUACIÓN DE LOS COSTOS AMBIENTALES EN LA UNIDAD EMPRESARIAL DE BASE CENTRAL AZUCARERO "ARGEO MARTÍNEZ" DE GUANTÁNAMO.

Cueva, A., Martínez, E. y Cobos, C. (2019). Los costos ambientales del crecimiento empresarial. Sur Academia: Revista Académica-Investigativa De La Facultad Jurídica, Social Y Administrativa, 4(8), 1-14. Recuperado de https://revistas.unl.edu.ec/index.php/suracademia/article/view/517

Ferreira-Sardinha, L., Pérez, M. y Vilariño, C. (2018). Indicadores de costos logísticos ambientales en cadena suministros de combustibles y lubricantes. Ciencias Holguín, 24(2), 1-12. Recuperado de http://www.ciencias.holguin.cu/index.php/cienciasholguin/article/view/1084/1166

Hansen, D. y Mowen, M. (2007). Administración de costos: contabilidad y control. México: Cengage Learning. Recuperado de http:/fullseguridad.net/wpcontent/uploads/2016/11/Administracion-de-costos.-Contabilidad-y-control-Hansen-5th.pdf

Lemos, J. y Rodríguez, J. (2016). Propuesta de diseño de costos ambientales para el proceso productivo del ácido cítrico de la empresa del ramo de alimentos de la ciudad de Palmira (valle). Contexto, 5, 183-196. DOI 10.18634/ctxj.5v.0i.661

López, A. (2019). La contabilidad de gestión ambiental como herramienta para generar sostenibilidad empresarial. Revista Investigación y Negocios, 12(19), 8-21. Recuperado de http://www.scielo.org.bo/scielo.php?script=sci_arttext\&pid=S2521-

$27372019000100003 \& \operatorname{lng}=e n \& n r m=i s o$

Mastrapa, B. y Sánchez, A. (2017). Sistema integrado de costos de calidad y medio ambiente para la gestión y la sostenibilidad empresarial. Retos de la Dirección, 11(1), 21-37. Recuperado de http://scielo.sld.cu/scielo.php?script=sci_arttext\&pid=S23061552017000100003

Maura, A. y Febles, J. (2018). Una aproximación a los costos ambientales en los suelos ferralíticos rojos para el logro de la sostenibilidad. Cofin Habana, 12(1), 192-208. Recuperado de http://scielo.sld.cu/scielo.php?script=sci_arttext\&pid=S2073-60612018000100013

Noguera, J. (2016). Costos ambientales. Recuperado de https://es.slideshare.net/InformacinVirtual/costos-ambientales-66532453

Parodi, P. (2010). Gestión de los costos ambientales en la actividad minera. Foro Virtual de Contabilidad Ambiental y Social. Buenos Aires, Argentina. Recuperado de 
IDENTIFICACIÓN Y EVALUACIÓN DE LOS COSTOS AMBIENTALES EN LA UNIDAD EMPRESARIAL DE BASE CENTRAL AZUCARERO "ARGEO MARTÍNEZ" DE GUANTÁNAMO.

http://www.econ.uba.ar/www/institutos/secretaradeinv/ForoContabilidadAmbiental/trabaj os2010/T_Parodi_Costos_Ambientales_Minera.pdf.

Salas, H. (2015). Integración de la dimensión ambiental al sistema de información financiero de empresas ubicadas en ecosistemas frágiles. Revista Universidad y Sociedad, 7(1), 102109. Recuperado de http://scielo.sld.cu/pdf/rus/v7n1/rus14115.pdf

Sánchez, V. y Bifaretti, M. (2008). Industria azucarera: ¿es el marco adecuado para lograr oportunidades de ecoeficiencia? Recuperado de https://es.scribd.com/document/156856091/c12

Sinforoso, S. (2018). Procedimiento metodológico para el reconocimiento de las externalidades ambientales en el costo de producción de las empresas cafetaleras veracruzanas (Tesis doctoral). Facultad de Contaduría y Administración, Universidad Veracruzana, Veracruz, México.

Sinforoso, S., Álvarez, E. y Vázquez, B. (2019). La integración del impacto ambiental del agua en la información financiera. Una mirada del sector hotelero de Tuxpan, Veracruz. Hitos de Ciencias Económico Administrativas, 25(73), 403-415. Recuperado de https://revistas.ujat.mx/index.php/hitos/article/view/3629/2774 